\title{
Early post-treatment with 9-cis retinoic acid reduces neurodegeneration of dopaminergic neurons in a rat model of Parkinson's disease
}

\author{
Lian-Hu Yin ${ }^{1,3}$, Hui Shen ${ }^{1}$, Oscar Diaz-Ruiz², Cristina M Bäckman², Eunkyung Bae', Seong-Jin Yu ${ }^{1 \dagger}$ and Yun Wang ${ }^{1 * \dagger}$
}

\begin{abstract}
Background: Retinoic acid (RA) is a biologically active derivative of vitamin A. Previous studies have demonstrated that RA has protective effects against damage caused by $\mathrm{H}_{2} \mathrm{O}_{2}$ or oxygen-glucose deprivation in mesangial and PC12 cells. Pretreatment with 9-cis-retinoic acid (9CRA) reduced infarction and TUNEL labeling in cerebral cortex as well as attenuated neurological deficits after distal middle cerebral artery occlusion in rats. The purpose of this study was to examine a protective role of 9cRA in dopaminergic (DA) neurons in a typical rodent model of Parkinson's disease (PD).

Results: The protective role of 9cRA was first examined in rat primary ventromesencephalic culture. Treatment with 9cRA significantly reduced 6-hydroxydopamine (6-OHDA)-mediated cell death and TUNEL labeling in cultured dopaminergic neurons. The protective effect was also examined in adult male rats. Animals received unilateral 6-OHDA lesioning at the left medial forebrain bundle on day 0 . Methamphetamine -induced rotational behavior was examined on days 6, 20 and 30 after lesioning. Animals were separated into 2 groups to balance rotational behavior and lesion extent on day 6 and were treated with either 9cRA or vehicle (i.c.v. on day $7+$ intra-nasal from day 8 to day 14). Post-treatment with 9cRA significantly reduced methamphetamine -mediated ipislateral rotation at 20 and 30 days after lesioning. In vivo voltammetry was used to examine DA overflow in striatum. Treatment with 9cRA significantly increased $\mathrm{KCl}$-evoked DA release in the lesioned striatum. 9cRA also increased tyrosine hydroxylase $(+)$ cell number in the lesioned nigra as determined by unbiased stereology.

Conclusion: Our data suggests that early post-treatment with 9cRA has a protective effect against neurodegeneration in nigrostriatal DA neurons in an animal model of PD.
\end{abstract}

\section{Background}

Retinoic acid (RA) is a biologically active derivative of vitamin A. Two major isoforms of RA, all-trans RA (atRA) and 9-cis RA (9cRA), have been identified. atRA is normally present at high levels in the developing spinal cord and at low levels in the forebrain of mouse embryos (Horton and Maden, 1995). On the other hand, 9cRA is not present in mouse embryos [1-3] or tissue extracts from adult rats [4]. RAs interact with two major groups of nuclear receptors: retinoic acid receptors (RAR) and

\footnotetext{
* Correspondence: ywang@intra.nida.nih.gov

${ }^{\dagger}$ Equal contributors

${ }^{1}$ Neural Protection and Regeneration Section, Intramural Research Program, National Institute on Drug Abuse, Baltimore, MD, USA

Full list of author information is available at the end of the article
}

retinoid $\mathrm{X}$ receptors (RXR). 9cRA binds with high affinity to RXR [5], whereas both 9cRA and atRA activate RAR [6,7].

RA has been reported to have protective effects after injury. RA inhibited $\mathrm{H}_{2} \mathrm{O}_{2}$-induced apoptosis via suppression of c-fos/c-jun expression and JNK activation in mesangial cells [8] and increased survival during anoxia/ glucose deprivation in PC12 cells [9]. These data suggest that RA can induce protective responses in cultured cells. The function of RA in the mature nervous system is less documented. Pretreatment with docosahexaenoic acid (DHA), a candidate ligand for RXR [10], reduced cerebral infarction induced by middle cerebral artery occlusion [11,12]. We previously reported that 9cRA is more potent than atRA in reducing ischemia -mediated brain infarction [13] and, furthermore, pretreatment with 9cRA attenuated DNA fragmentation in the

\section{Biomed Central}

(c) 2012 Yin et al.; licensee BioMed Central Ltd. This is an Open Access article distributed under the terms of the Creative Commons Attribution License (http://creativecommons.org/licenses/by/2.0), which permits unrestricted use, distribution, and reproduction in any medium, provided the original work is properly cited. 
lesioned cerebral cortex of stroke rats [14]. Taken together, these data suggest that 9cRA is neuroprotective against cerebral ischemia in vivo.

Several reports also indicate that RA can reduce degeneration induced by dopaminergic neurotoxins in culture. RA attenuates 6-hydroxydopamine (6-OHDA) and 1-methyl-4-phenylpyridinium (MPP+) -mediated neurotoxicity in SY5Y neuroblastoma cells [15]. The protective effects of RA in these cells involves the Akt pathway, increasing BCL2 while reducing p53 levels $[15,16]$. In rat midbrain slice cultures, treatment with a RAR agonist AM80 prevents IFN-r/LPS -induced dopaminergic cell loss [17]. These data suggest that RA has protective effects in dopaminergic neurons in culture. One recent study has shown that systemic administration of a high dose of RA ( $1 \mathrm{mg} / \mathrm{kg}$, i.p.) for 15 days reversed rotenone -induced impairment of locomotor activity without significantly reversing the reduction of dopamine (DA) in striatum [18]. In most of these studies, atRA was used to examine the protective effect of RA against dopaminergic neurotoxins. The protective role of 9cRA in dopaminergic neurons in an established model of PD has not been reported.

9cRA has also been shown to induce protection in animal model of stroke [13,14]. Application of a similar dose of atRA did not reduce the size of infarction after middle cerebral artery occlusion in rats, suggesting that 9cRA is more potent than atRA in neuroprotection [13]. Unlike atRA, 9cRA regulates the expression of proteins in the transforming growth factor (TGF) - $\beta$ superfamily. 9cRA enhances production of glial cell line derived neurotrophic factor (GDNF), and bone morphogenetic protein-7 (BMP-7) protein [14], in human neuroblastoma cells and U2 OS cells, respectively. 9cRA also increases midkin mRNA in primary cortical neurons [13]. All these trophic factors or proteins have been reported to reduce stroke insults $[13,19,20]$.

We previously demonstrated that intracerebroventricular administration of 9cRA time-dependently upregulates BMP7 mRNA expression in rodent brain [14]. The 9cRA - mediated reduction in cerebral infarction in stroke rats was significantly antagonized by the BMP antagonist noggin [14], suggesting that 9cRA induces protection through BMP7. Interestingly, BMP7 has been shown to reduce dopaminergic neurotoxins -mediated neurodegeneration in vivo and in vitro. BMP7 significantly reduced rotational behavior, partially preserved $\mathrm{KCl}$ - induced DA release in the lesioned striatum, and increased tyrosine hydroxylase $(\mathrm{TH})$ immunoreactivity in lesioned nigra in unilaterally 6-OHDA -lesioned rats [21]. Similarly, BMP7 antagonized high dose methamphetamine -induced DA cell loss in cultured dopaminergic neurons and striatal $\mathrm{TH}$ fiber density reduction in vivo [22]. Knocking down BMP7 or BMPRII receptors expression in mice increased sensitivity to dopaminergic neurotoxins in nigrostriatal dopaminergic neurons $[22,23]$. These data suggest that BMP7 has protective effects against DA-ergic neurotoxin -mediated degeneration. Since 9cRA enhances BMP7 expression, we reasoned it may also induce protection against 6-OHDA lesioning in an animal model of Parkinson's disease (PD) through the BMP7 pathway.

In this study, we examined the protective effect of 9cRA in a rodent animal model of PD. 9cRA was given at low dose from 7 days after unilateral 6-OHDA lesioning in adult rats. We found that post-treatment with 9cRA significantly reduced rotational behavior while increased the survival of dopamine cells in the lesioned nigra and $\mathrm{KCl}$-evoked DA release in lesioned striatum. Our data suggest that 9cRA induces neuroprotection against neurodegeneration in nigrostriatal DA neurons in an animal model of PD.

\section{Results}

\section{VM cell cultures}

We first examined protective effects of 9cRA in ventromesenphalic (VM) neuronal culture. Cells were fixed at 24 hours after 6-OHDA treatment. Typical TH immunoreactivity of VM cultured cells is demonstrated in Figure 1A. Treatment with 6-OHDA dose-dependently reduced $\mathrm{TH}(+)$ cell density (Figure $1 \mathrm{~B}, \mathrm{p}<0.001$, two way ANOVA). 9cRA or vehicle was given at 2 hours after addition of 6-OHDA or vehicle to the well. Treatment with 9cRA $(50 \mathrm{nM})$ did not alter TH cell density in non-lesioned cells; however, 9cRA significantly attenuated 6-OHDA-medated loss of TH cells at 24 hours after lesioning (Figure 1B, $\mathrm{p}=0.022$, two way ANOVA). We also found that 6-OHDA treatment enhanced the density of TUNEL labeling. 9cRA significantly reduced 6-OHDA -mediated TUNEL activity (Figure $1 \mathrm{C}$ and D, $\mathrm{p}<0.001$, one way ANOVA + Newman-Keuls test).

We previously demonstrated that 9cRA is more potent than atRA against ischemic brain injury in vivo. Similarly, we found that 9cRA is more efficient that atRA against 6-OHDA -mediated neurodegeneration in VM TH neurons. More TH neurons survived post-treated with 9cRA, compared to atRA, after 6-OHDA lesioning (Figure 2, $\mathrm{p}<0.001, \mathrm{t}$ test).

\section{Animal study}

\section{(A) Rotation}

A total of 17 rats received unilateral 6-OHDA lesioning on day 0 . Methamphetamine -induced rotational behavior was examined on days 6, 20 and 30 after 6-OHDA lesioning. Animals were separated into 2 groups (veh and 9cRA) to equalize group rotational behavior and thus lesion extent on day 6 . Two animals were excluded because the rotation was higher than 960 turns/hour to avoid maximal 


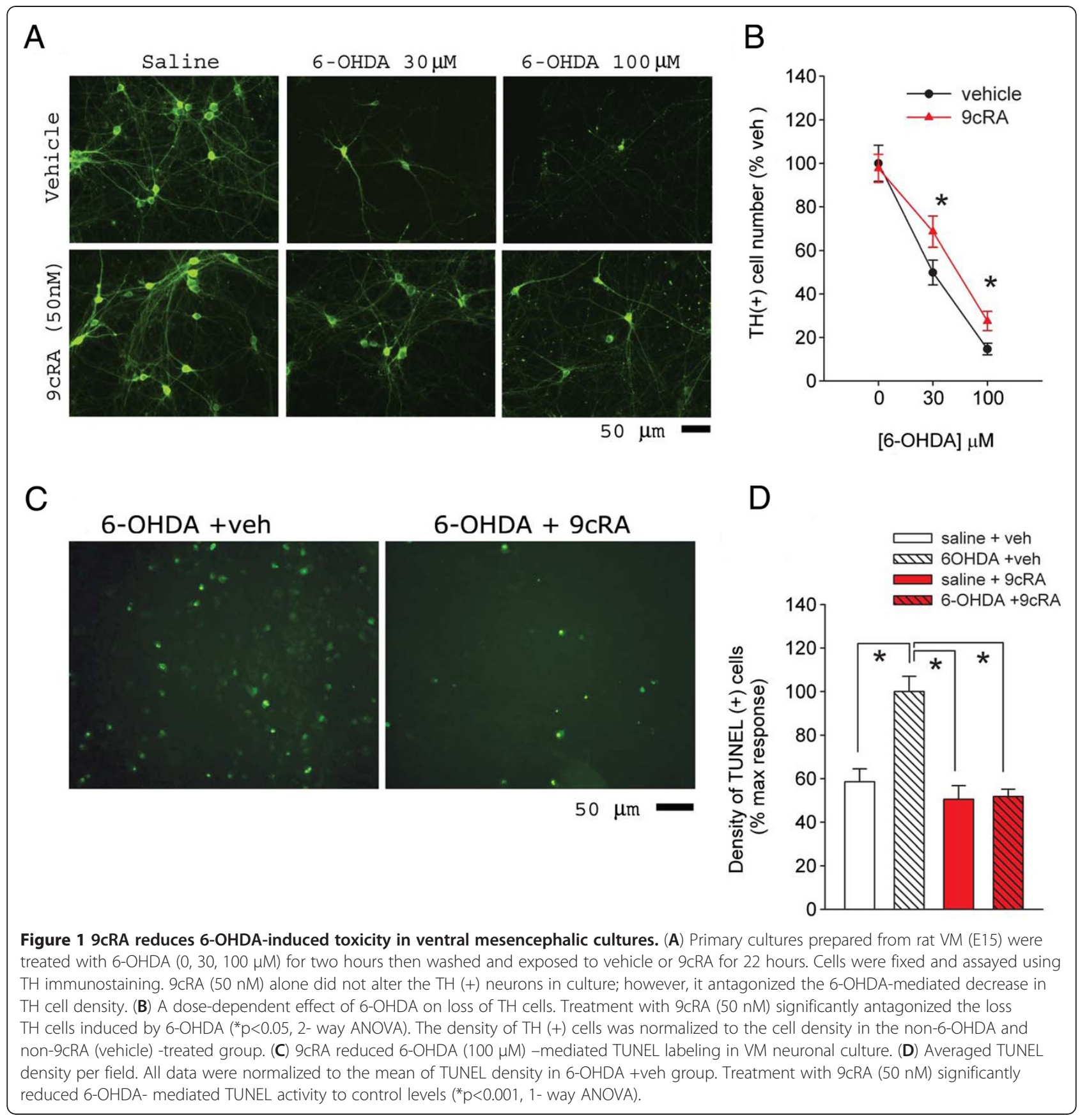

lesioning before neural protective treatment. There was no difference in rotation prior to 9cRA or vehicle treatment between these two groups $(\mathrm{p}=0.789$, $\mathrm{t}$ test). Averaged rotation (turns/hour) was $494.1+/-73.6$ (for RA group, $n=7$ ) and $523.6+/-77.6$ (for veh group, $n=8$ ). Animals were treated with 9cRA or veh from day 7 to day 14 (Figure 3). The rotational behavior was re-examined on days 20 and 30 after lesioning (Figure 3). Using a two way ANOVA, we determined that 9cRA, compared to vehicle, significantly reduced methamphetamine -mediated rotation in the unilaterally 6-OHDA-lesioned rats $(\mathrm{p}=0.028$, Figure 3).

\section{(B) $\mathrm{KCl}$-induced $\mathrm{DA}$ release and $\mathrm{DA}$ clearance in striatum}

$\mathrm{KCl}$-evoked DA release in striatum was examined using in vivo voltammetry in 11 rats at $>2$ months after unilateral 6-OHDA injection. Five of these animals were treated with 9cRA and the other six were treated with vehicle. $\mathrm{KCl}$-evoked DA release was recorded in 143 striatal sites between $3.5 \mathrm{~mm}$ to $7.0 \mathrm{~mm}$ below the brain surface and 


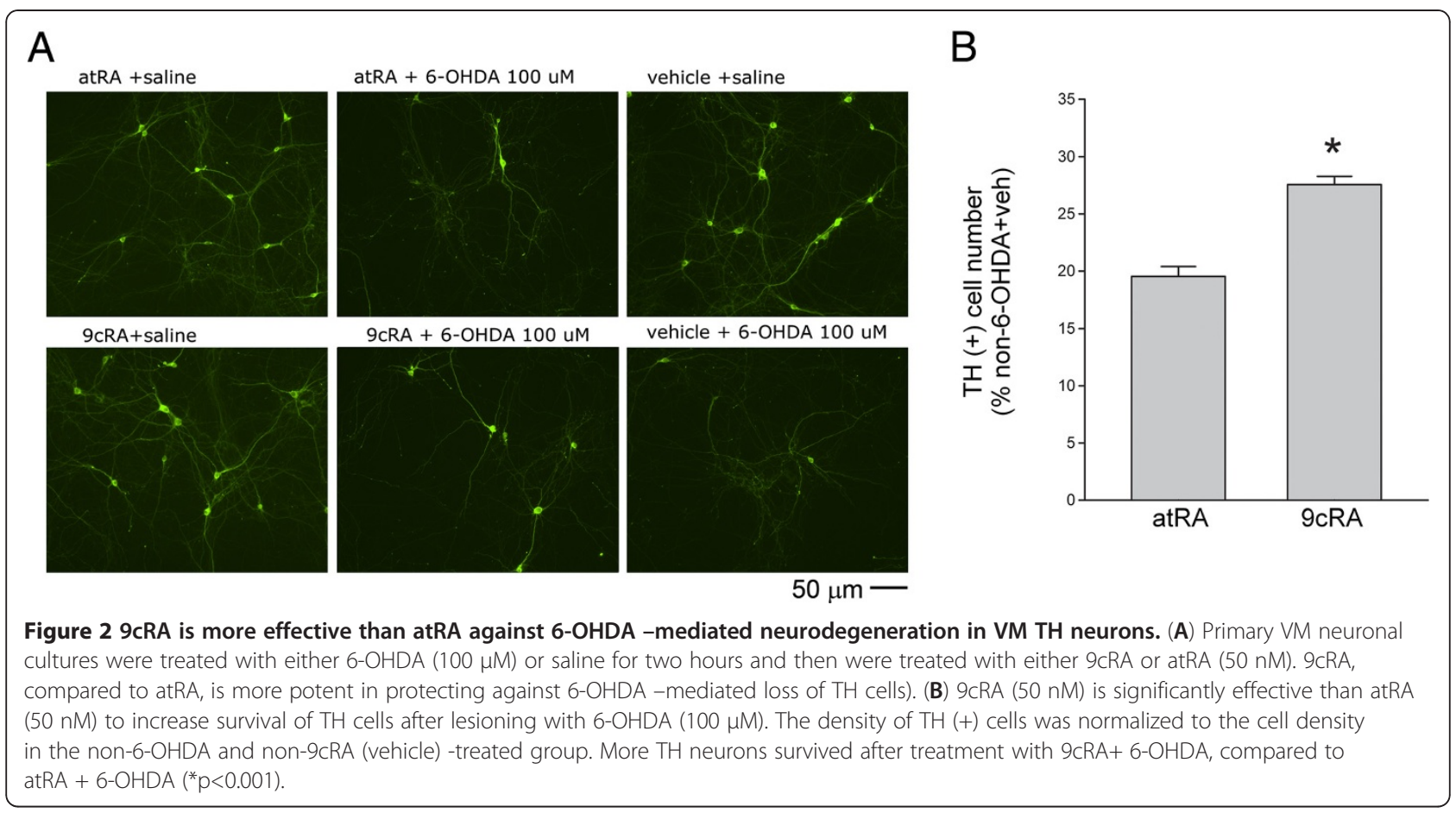

2.0-2.5 mm lateral, $0-1 \mathrm{~mm}$ anterior to bregma. Of these, 80 sites were taken from the striatum ipsilateral (L) to the 6-OHDA injection while 44 sites were recorded in the contralateral ( $\mathrm{R}$ ) striatum. Average dose of $\mathrm{KCl}$ ejected from micropipette was $204.0 \pm 9.1 \mathrm{nl}$ per site. Local application of $\mathrm{KCl}$ resulted in release of dopamine in all sites in non-lesioned (R) striatum in both 9cRA and vehicle

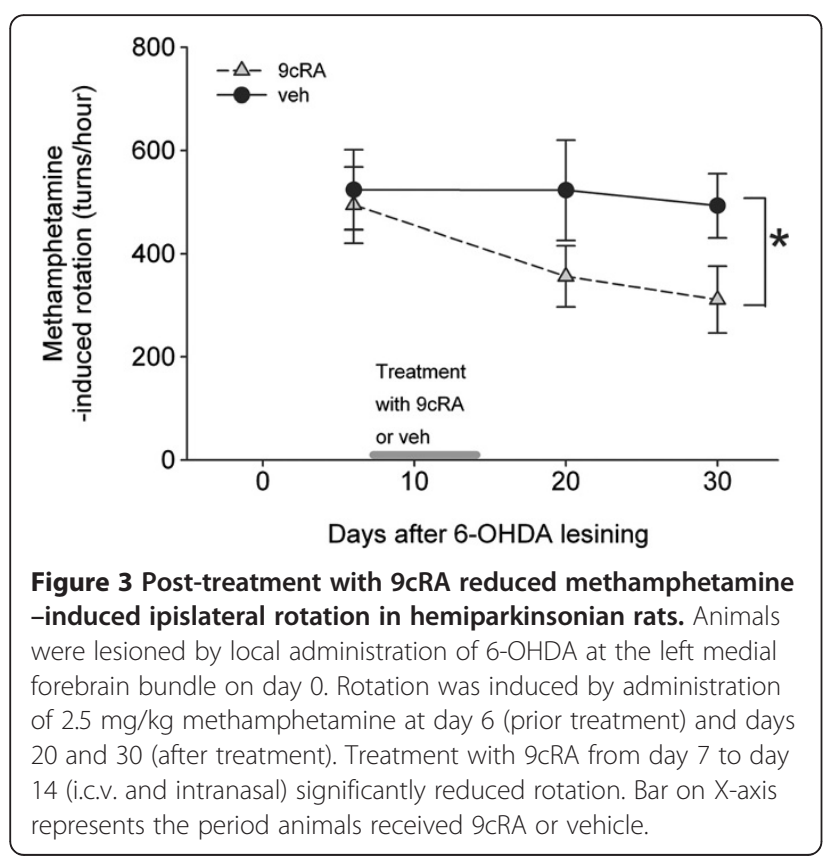

-treated mice. On the other hand, the peak of DA release was reduced in the lesioned striatum. Typical extracellular dopamine tracings from left and right striatum are shown in Figure 4.

Previous voltammetric studies have shown a dose response relationship between the peak of extracellular DA level and log dose of applied DA through a micropipette in rat striatum $[24,25]$. In this study, the amplitude of DA release was thus normalized by comparison to the log volume (in $\mathrm{nL}$ ) of $\mathrm{KCl}$ used. A similar approach has been used in our previous papers [26,27]. There is a significant reduction in $\mathrm{KCl}$-evoked DA release in the lesioned striatum, compared to the nonlesioned striatum (Figure 5, $\mathrm{p}<0.001$, one way ANOVA; $\mathrm{p}<0.001)$ in both vehicle and 9cRA treated animals $(\mathrm{p}<0.001$, post-hoc Newman Keuls test). However, treatment with 9cRA significantly increased $\mathrm{KCl}$-evoked DA release in the lesioned side striatum $(\mathrm{p}=0.041$; Figure 5A), compared to vehicle. No difference was found in the non-lesioned side striatum between 9cRA and vehicle -treated animals $(\mathrm{p}=0.263)$.

The rate of DA clearance ( $\mathrm{nM}$ per sec) after $\mathrm{KCl}$ application was calculated by measuring the slope between T20 and T60 (Figure 4A) of the DA currents, as described previously $[27,28]$. There was a significant reduction of DA clearance in the lesioned side striatum (Figure 5B, $\mathrm{p}<0.001$, one way ANOVA; $\mathrm{p}<0.001$, posthoc Fisher LSD test). Treatment with 9cRA significantly increased DA clearance rate in the lesioned side striatum $(\mathrm{p}=0.028$; Figure 5B) compared to vehicle. 

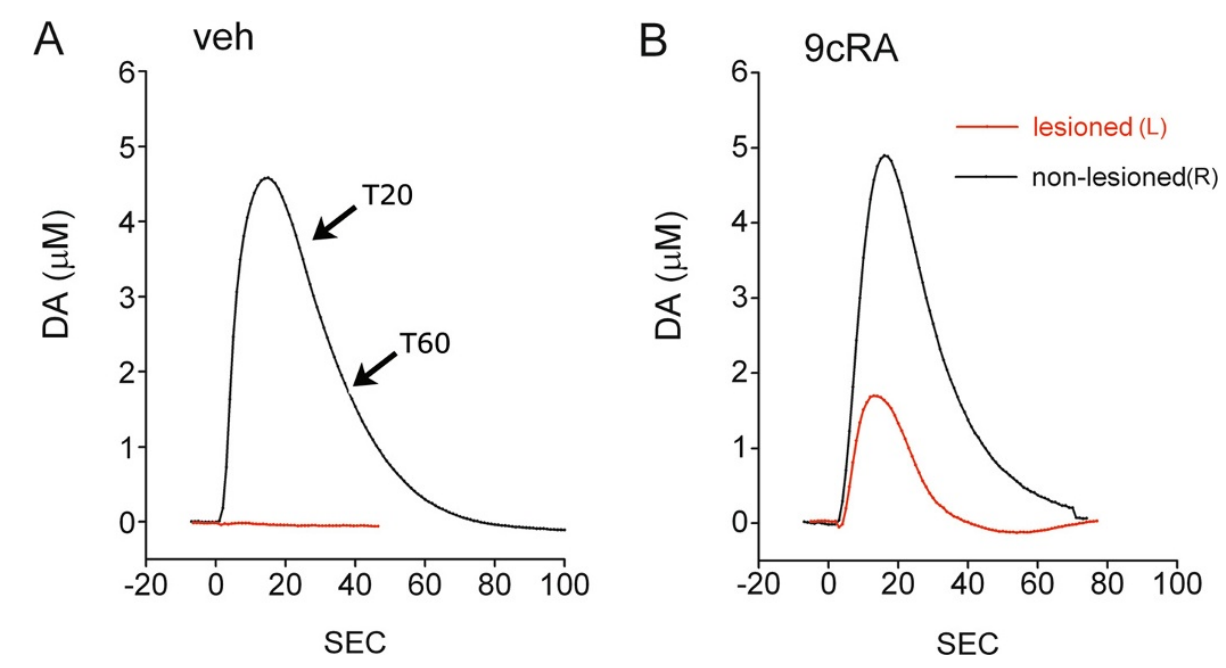

Figure 4 Typical voltammetric tracings of extracelluar DA concentration in dorsal striatum in unilaterally 6-OHDA lesioned rats.

Examples of $\mathrm{KCl}$-evoked dopamine release were taken from dorsal striatum (3.5 to $5 \mathrm{~mm}$ below brain surface). $\mathrm{KCl}$ ( $150-200 \mathrm{~nL} \times 70 \mathrm{mM}$ ) was delivered locally through a micropipette next to DA sensor at time 0 . (A) Local administration of KCl induced DA release in non-lesioned striatum (black tracing) in a vehicle treated animal. In the same animal, $\mathrm{KCl}$-mediated DA release was almost abolished in lesioned striatum (red tracing). (B) In contrast, 9cRA treatment partially restored $\mathrm{KCl}$-induced DA release in lesioned striatum in another animal. 9cRA did not alter DA release in the non-lesioned striatum. Note that slope between T20 and T60 (A) is used to calculate the rate of clearance in Figure 5B.

\section{(C) TH immunoreactivity in nigra}

$\mathrm{TH}$ immunoreactivity was studied at $>2$ months after unilateral 6-OHDA lesioning, TH-positive [TH (+)] cell number in SN and in VTA were counted in 11 rats. Typical TH immunostaining of 6-OHDA -lesioned animals receiving either 9cRA or vehicle treatment is shown in Figure 6. Injection of 6-OHDA consistently reduced $\mathrm{TH}(+)$ cell number in $\mathrm{SN}$ in either vehicle or 9cRA -treated animals $(\mathrm{p}<0.001,2$-Way ANOVA; $\mathrm{p}<0.001$, post-hoc Newman Keuls test, Figure 7A). Treatment with 9cRA significantly increased $\mathrm{TH}(+)$ cell number in the lesioned side SN ( $\mathrm{p}=0.026$, post-hoc Newman Keuls test) compared to vehicle. No change was found in the contralateral (non-lesioned) side SN $(p=0.432)$. Although there is a trend that A10 TH cell number can be suppressed by 6-OHDA lesioning, there is no significant difference after lesioning or after 9cRA treatment (Figure 7B, $\mathrm{p}=0.734$ ).

\section{Discussion}

In this study, we found that density of $\mathrm{TH}(+)$ neurons and fibers in primary VM cultures were dose dependently reduced at 2 days after 6-hydroxydopamine treatment. 6-OHDA also induced DNA fragmentation as demonstrated in TUNEL labeling. These data are in agreement with our previous studies and suggest that administration of 6-OHDA causes activation of apoptotic pathways that lead to the death of VM dopaminergic neurons. We found that early and post treatment with 9cRA reduced 6-OHDA -mediated injury in VM cells. 6-OHDA -mediated reduction of TH cell density was significantly antagonized by 9cRA, suggesting that 9cRA is neuroprotective against 6-OHDA -mediated neurodegeneration in dopaminergic neurons in vitro.

Previous studies have demonstrated that RA exerts anti-apoptotic and antioxidant activity in neuronal and kidney cells. RA reduced staurosporine-induced oxidative stress and apoptosis by preventing the decrease in the levels of $\mathrm{Cu}$-, $\mathrm{Zn}$-superoxide dismutase (SOD-1) and $\mathrm{Mn}$-superoxide dismutase (SOD-2) in primary hippocampal cultures [29] and by facilitating NGF-induced protection in chick embryonic neurons [30]. RA suppressed hydrogen peroxide -induced apoptotic nuclear condensation and membrane blebbing in rat glomeruli mesangial cells. Our recent studies also indicated that 9cRA reduced the density of TUNEL labeling, a marker for apoptosis/necrosis in ischemic cortex after middle cerebral artery occlusion in rats [14]. In this study, we demonstrated that treatment with 9cRA reduced 6OHDA-mediated DNA fragmentation in culture. Taken together, our data suggest that 9cRA inhibits apoptosis and/or necrosis induced by 6-OHDA.

Using an established rodent model of PD, we also demonstrated the protective effect of 9cRA in vivo. Administration of amphetamine analogs causes ipislateral rotation in unilaterally 6-OHDA -lesioned rats due to differential increase of dopaminergic activity on the intact side. There is a correlation between amphetamineinduced ipislateral rotation and the depletion of dopamine in the nigra [31]. In this study, 9cRA was given after rotation experiment on day 6 post lesioning. We separated 

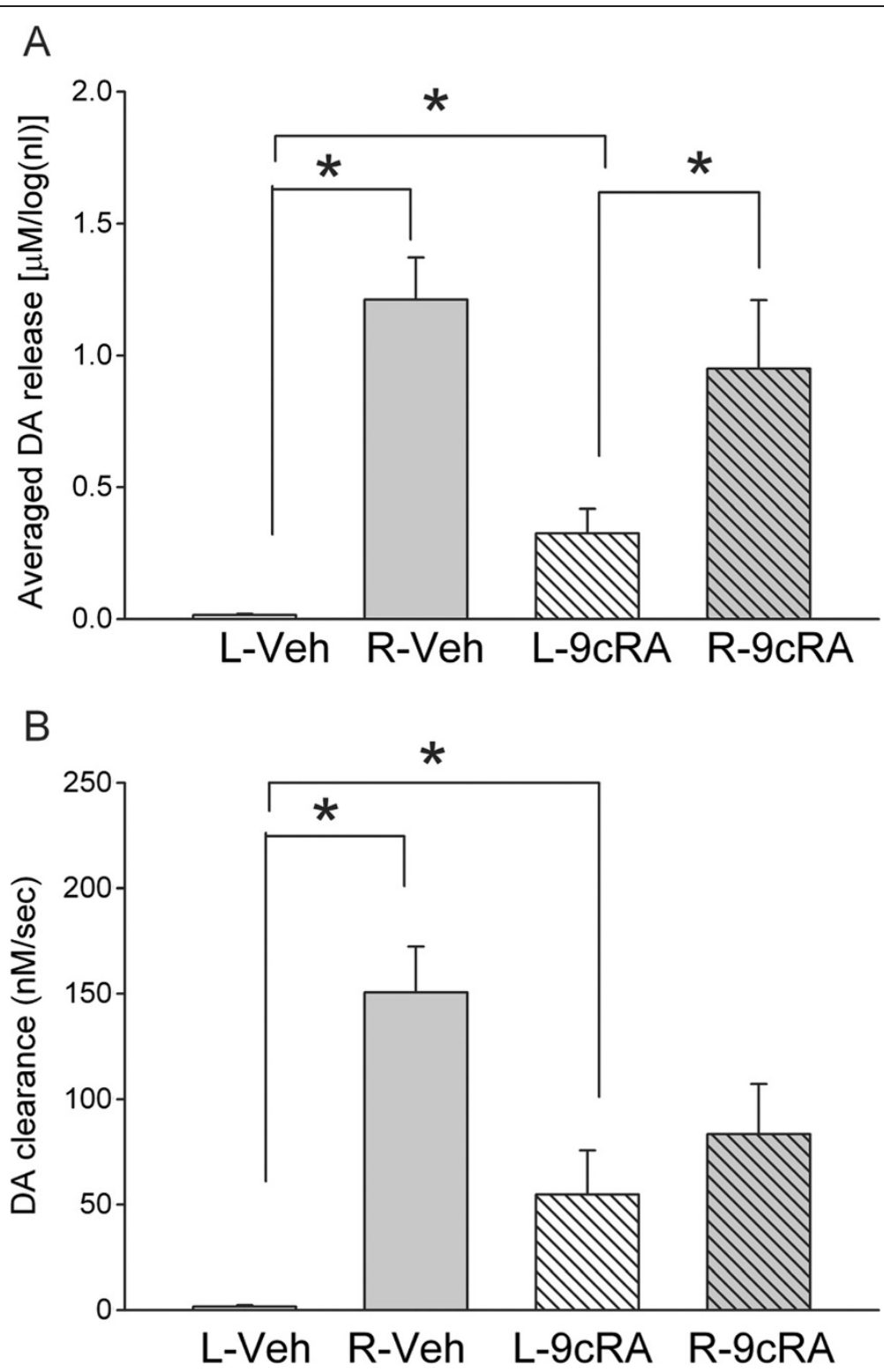

Figure 5 Averaged DA release (A) and rate of DA clearance (B) in striatum in 9cRA or vehicle treated hemiparkinsonian rats. (A) The peak amplitude of the DA signal, induced by local $\mathrm{KCl}$ administration, was averaged in lesioned ( $\mathrm{L}$, left) or non-lesioned ( $\mathrm{R}$, right) striatum of unilaterally 6-OHDA -lesioned rats. KCl-evoked DA release was almost abolished in the $\mathrm{L}$ striatum in vehicle treated rats. Treatment with 9cRA significantly increased DA release in the lesioned striatum. (B) The rate of DA clearance $(\mathrm{nM} / \mathrm{s})$ after $\mathrm{KCl}$ application was calculated between T20 and T60. There is a significant reduction in DA clearance in the striatum ipsilateral to the 6-OHDA lesioning in vehicle treated rats. 9cRA treatment significantly enhanced rate of DA clearance.

animals to 2 groups to balance rotational behavior before treatment; the difference in lesioning prior to treatment was thus minimal between two groups. We demonstrated that post-treatment with 9cRA, compared to vehicle, significantly attenuated rotation. Our data support that early and post-treatment with 9cRA reduced behavioral imbalance in hemiparkinsonian rats.

The protective effect of 9cRA is further supported by in vivo electrochemical data at 2 months after lesioning. We used high speed chronoamperometry to examine the time course of $\mathrm{KCl}$-evoked DA release and clearance in striatum. The dose of $\mathrm{KCl}$ applied locally was between $7-14 \times 10^{-11}$ mole $(70 \mathrm{mM} \times 204.0 \pm 9.1 \mathrm{nl})$ per site The dose, concentration and volume of the $\mathrm{KCl}$ solution have been previously reported to induce depolarization and release of dopamine at dopaminergic nerve terminals in vivo [26,32]. We found that local administration of $\mathrm{KCl}$ induced DA release equally in the non-lesioned side striatum in 9cRA or vehicle -treated rats. In the lesioned side striatum, $\mathrm{KCl}$-evoked $\mathrm{DA}$ release and the 


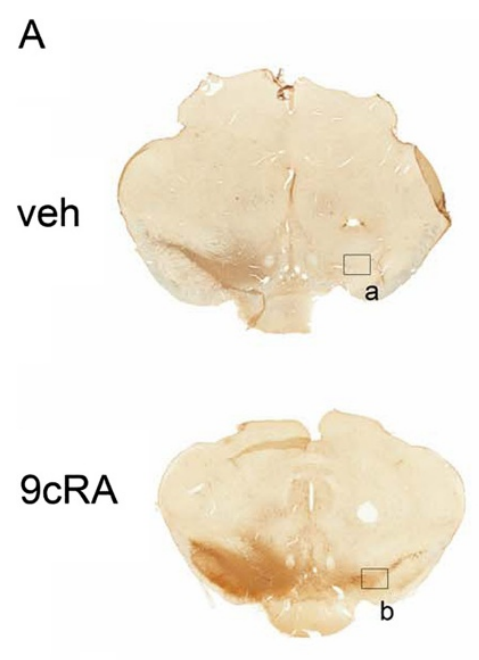

B a

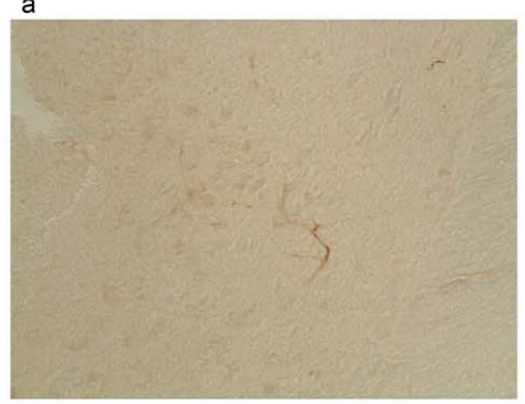

c

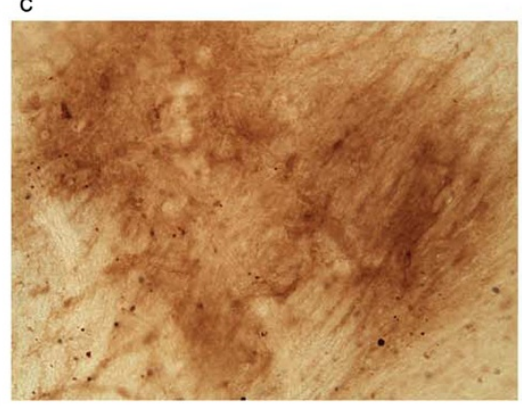

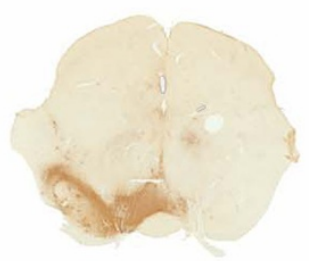
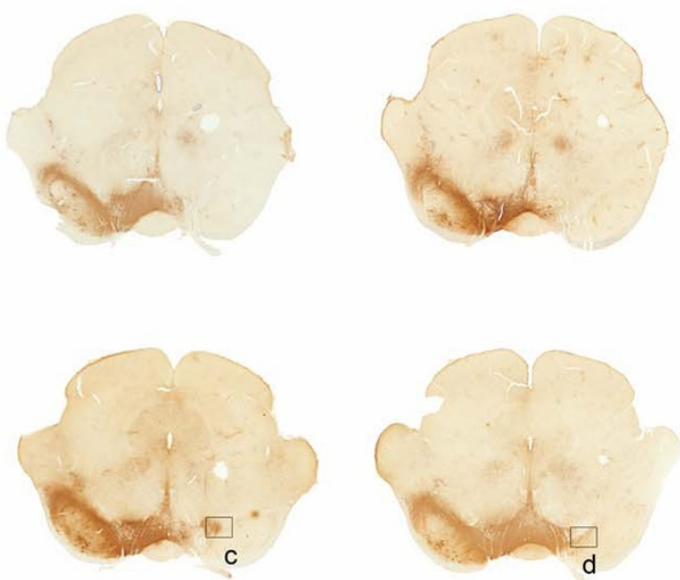

b

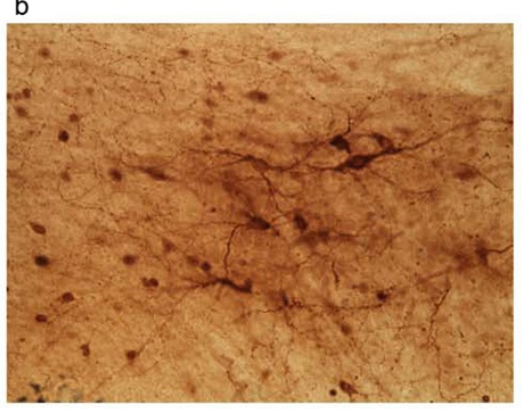

d

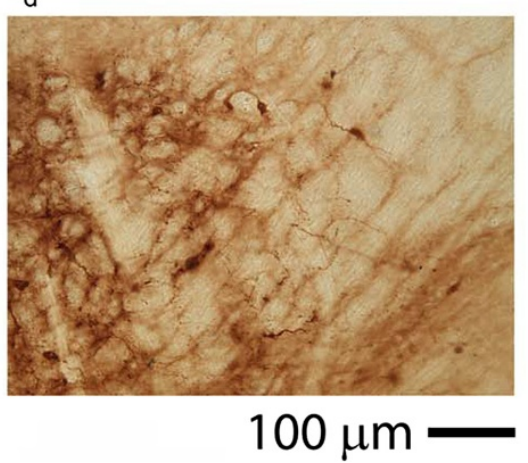

Figure 6 Post-treatment with 9cRA attenuated the loss of TH cells in nigra. TH immunoreactivity was conducted at $>2$ months after unilateral 6-OHDA lesioning. (A) Almost no TH activity was found in the lesioned side nigra (left panel, rostral nigra; middle and right panels, caudal nigra) in 3 vehicle treated animals. Treatment with 9cRA partially blocked the loss of TH immunoreactivity in another 3 lesioned rats. (B) Labels in each panel correspond to the blocks at higher magnification in (A). Note that almost no TH cells in lesioned SN in the vehicle treated rat (B-a). In contrast, TH $(+)$ cells were found in rostral $(\mathbf{B}-\mathbf{b})$ and caudal $(\mathbf{B}-\mathbf{c}, \mathbf{d}) \mathrm{SN}$ in the lesioned rats treated with 9cRA.

rate of DA clearance were greatly diminished in animals receiving vehicle treatment. Treatment with 9cRA did not induce a full recovery but significantly increased $\mathrm{KCl}$-evoked DA release and DA clearance in the lesioned side striatum. These electrochemical data support that post-treatment with 9cRA protects against 6-OHDA -mediated deficiency in DA function in striatum.

Using unbiased stereology, we demonstrated that posttreatment with 9cRA significantly reduced the loss of
$\mathrm{TH}$ cells in nigra. In contrast to a $95 \%$ reduction in $\mathrm{TH}$ cells in the lesion side nigra in animals with vehicle treatment, 70\% TH (+) neurons were lost in the lesioned animals with 9cRA treatment. Similarly, there was about 99\% reduction in $\mathrm{KCl}$-evoked DA release in striatum in vehicle -treated animals. The reduction in DA release was only $66 \%$ after $9 \mathrm{cRA}$ treatment. Taken together, our data suggest that 9cRA has a protective effect against 6OHDA injury in nigrostriatal dopaminergic neurons. It 

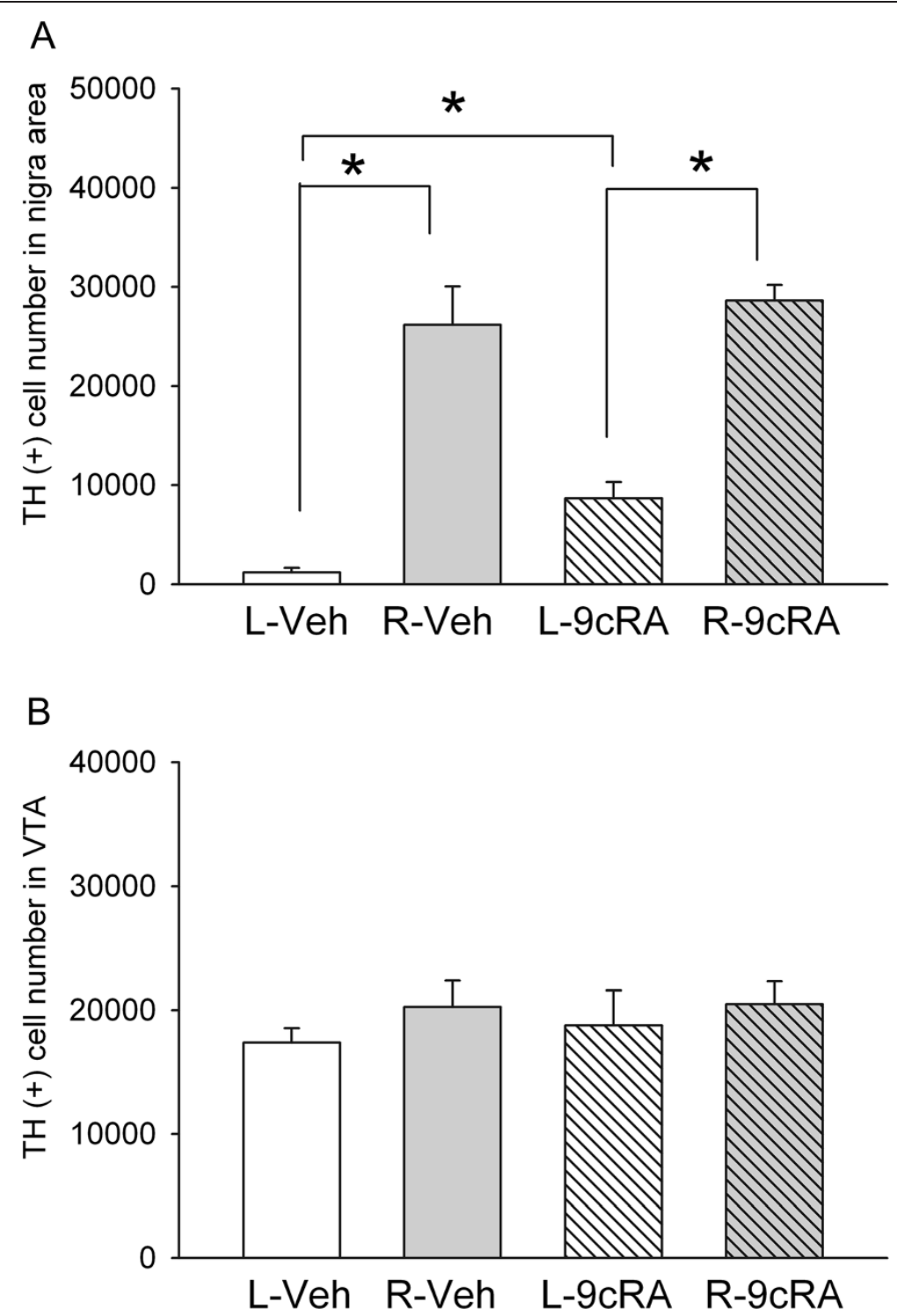

Figure 7 9cRA treatment increased survival of TH cells in lesioned nigra. TH $(+)$ cell number $(\mathbf{A})$ in SN and (B) in VTA were counted using unbiased stereology in 11 rats. (A) Injection of 6-OHDA reduced cell number of dopamine neurons in lesioned side $(L) S N(p<0.001)$. Treatment with 9cRA significantly increased dopamine cell number in the lesioned side ( $L$ ) $S N(p=0.026)$. No change was found in the contralateral (non-lesioned, R) side SN ( $p=0.432)$. (B) TH cell number in A10. There is no significant difference after lesioning or after 9cRA treatment in VTA $(p=0.734)$

has been reported that the symptoms of the PD appear when $70-80 \%$ dopamine is depleted in patients [33,34]. In this study, we demonstrated that early treatment with 9cRA can reduce the death of DA neurons to $70 \%$. It is thus possible that post-treatment with 9cRA can be beneficial to PD patients by slowing down its progressive neurodegeneration.

We previously demonstrated that 9cRA can selectively induce BMP7 mRNA expression in the brain. In this study, we demonstrated that 9cRA reduced 6-OHDA -mediated program cell death in culture. BMP7 has antiapoptotic properties. BMPs reduced caspase-3 activation and DNA fragmentation in the ischemic brain $[35,36]$ and attenuated dopaminergic neurotoxin -mediated apoptosis and cell death in nigrostriatal DA neurons. Interestingly, the reduction of TUNEL labeling by 9cRA in stroke brain was antagonized by the BMP antagonist noggin. Taken together, these data suggest that the protective effects of 9cRA may be indirectly mediated through a BMP signaling mechanism.

RA analogs, such as 9cRA and atRA, are agonists for RXR and RAR receptors. Compared to atRA, 9cRA is a more selective agonist for RXR. Although both atRA and 9cRA are neural protective against oxygen-glucose 
deprivation in hippocampal neurons [37], a differential sensitivity of these two ligands have been seen in other brain regions. Animals pretreated with 9cRA had lesser cortical infarction than those treated with atRA after middle cerebral artery occlusion [13]. Using RTPCR, we also found that 9cRA is more potent than atRA in inducing BMP7 (unpublished observation) and midkine [13] expression in primary cortical cultures. We demonstrated here that 9cRA is more potent than atRA, at 50 $\mathrm{nM}$, against 6-OHDA -mediated neurodegeneration in $\mathrm{TH}$ neuronal culture. Taken together, these differential responses of 9cRA suggest that neuroprotection induced by 9cRA involves activation of RXR.

Although 9cRA has a high affinity for RXR, it is not detectable in adult brain tissue and, thus, may not be a candidate endogenous ligand for this receptor. Studies have indicated that the polyunsaturated fatty acids, such as linolenic and docosahexaenoic acid, activate RXR $[10,38]$. These polyunsaturated fatty acids have also been reported to reduce neurodegeneration $[39,40]$. Future experiments are required to investigate the mechanism of protection induced by these polyunsaturated fatty acids and their effects on RXR in animal model of PD.

There are several limitations to deliver drug to brain days after onset of 6-OHDA lesioning. Drugs given systemically may not easily cross the blood brain barrier (BBB) and can be degraded through first pass metabolism. Intracerebral delivery is not feasible for repeated drug administration and may require chronic cannulation. Previous reports have indicated that small molecules can by-pass the BBB and reach brain parenchyma non-invasively through an intranasal delivery. In this study, 9cRA was given initially through i.c.v. and then repeatedly through an intra-nasal route. We found that intranasal administration of 9cRA increased brain 9cRA level to $3 \mathrm{ng} / \mathrm{g}$ or $10 \mathrm{nM}$ at 1 hour after delivery as detected by LC-MS/MS analysis (Additional file 1:Figure S1). No 9cRA was seen after vehicle treatment. Previous studies have shown that 9cRA can activate RXR receptor at this concentration [41].

In a preliminary study, we treated 6-OHDA -lesioned rats with intranasal 9cRA without i.c.v injection from day 7 to day 14 . We did not find significant behavioral improvement after intranasal 9cRA treatment and days 20 and 30, suggesting that a loading dose of 9cRA, given i.c.v. on day 7 is required for this protective reaction.

\section{Conclusion}

We have demonstrated that $9 \mathrm{cRA}$, given from 7 th day to 14th day post 6-OHDA lesioning, reduced rotational behavior and loss of TH cells in nigra, while increased DA release function in striatum. Our data suggest that 9cRA has neuroprotective effects against neurodegeneration in a rodent model of $\mathrm{PD}$. The protection may be more prominent if 9cRA is given earlier or prior to lesioning. The effectiveness of 9cRA may be clinically useful for patients at early stages of PD.

\section{Methods}

\section{Primary cultures of rat ventral mesencephalon}

Primary cultures were prepared from embryonic (E1415) ventral mesencephalon (VM) tissues obtained from fetuses of timed-pregnant Sprague-Dawley rats (Charles River Laboratories, Wilmington, MA), according to published procedures with some modification. The whole brain was removed aseptically and a small piece of tissue comprising the VM was dissected. After removing the blood vessels and meninges, pooled VM tissues were trypsinized (0.25\%; Invitrogen, Carlsbad, CA) with gentle mixing for $15 \mathrm{~min}$ at $37^{\circ} \mathrm{C}$. After rinsing off trypsin with pre-warmed DMEM/F-12 (Invitrogen), cells were dissociated by trituration, counted and plated into 96-well $\left(6.0 \times 10^{4} /\right.$ well $)$ cell culture plates pre-coated with polylysine (Sigma-Aldrich). The culture plating medium consisted of Dulbecco's modified Eagle medium/F12 supplemented with $10 \%$ heat-inactivated fetal bovine serum, $1 \mathrm{mM}$ L-glutamine and 2\% B27 (Invitrogen). Cultures were maintained at $37^{\circ} \mathrm{C}$ in a humidified atmosphere of $5 \% \mathrm{CO}_{2}$ and $95 \%$ air. The cultures were fed by exchanging $50 \%$ of media with feed media (Neurobasal medium, Invitrogen) with $2 \%$ B27 with antioxidants (+AO) supplement on DIV (days in vitro) 3 and 5. ON DIV7, cultures were fed with feed media containing B27 supplement without antioxidants ((-) AO, Invitrogen). Freshly made 6-hydroxydopamine (in $20 \mu \mathrm{M}$ ascorbic acid saline solution) or saline (with $20 \mu \mathrm{M}$ ascorbic acid) was added to the wells on DIV 10. After incubation for 2 hours, cultures were washed with (-)AO B27 3 times. RA or vehicle was added to the well at the last wash. Cells were returned to a $37^{\circ} \mathrm{C}$ incubator for 22 hours then fixed with $4 \%$ paraformaldehyde (PFA) for immunoreactivity.

\section{In vitro immunoreactivity and quantitation}

After removing PFA solution, cells were washed with PBS and the fixed cultures were treated for 1 hour with blocking solution (2\% BSA, 0. 1\% Triton X-100 and 5\% goat serum in PBS). The cells were then incubated for 2 days at $4^{\circ} \mathrm{C}$ with a mouse monoclonal antibody against $\mathrm{TH}$ (1:500; Chemicon, Temecula, CA, USA). The cells were then rinsed three times in PBS. The bound primary antibody was visualized using the AlexaFluor 488 goat anti-mouse secondary (Invitrogen). Images were acquired using a SPOT RT camera (Diagnostic Instruments, Inc., Sterling Heights, MI) attached to a NIKON TE2000 inverted microscope. $\mathrm{TH}+$ cells were manually counted in $10 \mathrm{x}$ images ( 4 fields per well of 96 well plate). All immunoreactive counts and quantitation were expressed as 
percentage of untreated cells. Experiments were repeated 2-3 times with $n=3-9$ wells per group per experiment.

\section{In vitro Terminal deoxynucleotidyl transferase (TdT)-mediated dNTP nick -end labeling (TUNEL)}

Cultures were assayed for DNA fragmentation using a TUNEL-based method (In Situ Cell Death Detection Kit; Roche, Indianapolis, IN). Briefly, 4\% PFA fixed cells were permeabilized in $0.1 \%$ Triton $\mathrm{X}-100$ in $0.1 \%$ sodium citrate for $2 \mathrm{~min}$ on ice. To label damaged nuclei, $50 \mu \mathrm{L}$ of the TUNEL reaction mixture was added to each sample and kept at $37{ }^{\circ} \mathrm{C}$ in a humidified chamber for $60 \mathrm{~min}$. Procedures for positive and negative controls were carried out as described in the manufacturer's manual (Roche). Controls consisted of not adding the label solution (terminal deoxynucleotidyl transferase) to the TUNEL reaction mixture. Material was examined using a Nikon TE2000 inverted microscope equipped with fluorescence. TUNEL(+) cells were manually counted in $20 \mathrm{x}$ images (4 fields per well of 96 well plate).

\section{Animals}

Adult male Sprague Dawley rats from Charles River Lab Inc. were used for this study. The use of animals was approved by the Animal Care and Use Committee, National Institute on Drug Abuse, IRP.

\section{6-hydroxydopamine lesioning}

Rats were anesthetized with chloral hydrate (400 $\mathrm{mg} / \mathrm{kg}$, i.p.) and placed in a stereotaxic frame. 6hydroxydopamine $(2.27 \mu \mathrm{g} / \mu \mathrm{l} \times 5 \mu \mathrm{l}$ in $0.9 \% \mathrm{NaCl}$ containing $0.2 \mathrm{mg} / \mathrm{ml}$ ascorbic acid) was unilaterally injected into the left medial forebrain bundle $(-4.4 \mathrm{~mm}$ AP, $1.2 \mathrm{~mm}$ ML relative to bregma and $8.4 \mathrm{~mm}$ below skull) over $4 \mathrm{~min}$. After injection, a piece of bone wax (W810, Ethicon) was applied to the burr hole in the skull to prevent efflux of the solution.

\section{Behavioral measurement}

Rotational behavior [42,43] was evaluated using a multichannel rotometer system (RotoMax, AccuScan Instruments, Inc). Six days after 6-OHDA lesioning, all animals were challenged with methamphetamine $(2.5$ $\mathrm{mg} / \mathrm{kg}$ ). Each animal was placed in a cylindrical test chamber for $90 \mathrm{~min}$. The highest consecutive net rotations (ipsilateral -contralateral rotation) over $60 \mathrm{~min}$ were used for analysis. Animals that rotated in excess of 300 turns/hour were selected for 9cRA or vehicle treatment. Methamphetamine -induced rotation was reexamined on 20 and 30 days after lesioning.

\section{Administration of 9cRA}

Animals were separated into 2 groups to receive 9cRA or vehicle after rotation was measured by rotometer on day 6 after 6-OHDA lesioning. On the $7^{\text {th }}$ day after lesioning, animals were anesthetized with chloral hydrate (0.4 g/kg, i.p.). 9cRA $(1 \mu \mathrm{g} / 1 \mu \mathrm{l} \times 20 \mu \mathrm{l}$, dissolved in $10 \%$ DMSO, Sigma, pH 7.0) or vehicle (10\% DMSO in saline $\mathrm{pH}$ 7.0, $20 \mu \mathrm{L}$ ) was given intracerebroventricularly through a Hamilton syringe. The coordinates for intracerebroventricular injections were: $0.8 \mathrm{~mm}$ posterior to the bregma, $1.5 \mathrm{~mm}$ lateral to the midline; $3.5 \mathrm{~mm}$ below dura surface. The needle was retained in place for 5 min after injection. After injection, a piece of bone wax (W810, Ethicon) was applied to the burr hole in the skull to prevent efflux of the solution. Animals were anesthetized with isoflurane for 5-10 min each day from day 8 to day 14 after lesioning and were placed in a supine position. 9cRA (conc $=1 \mu \mathrm{g} / 1 \mu \mathrm{l}$ ) or vehicle was delivered into nostrils of each rat at dose of $20 \mu \mathrm{l}$ daily as previously described [44]. No difference in body weight was found between animals treated with 9cRA or vehicle.

\section{$\mathrm{KCl}$-evoked DA release}

$\mathrm{KCl}$-evoked DA release in striatum was measured at 2 months after lesioning. Animals were anesthetized using urethane $(1.25 \mathrm{~g} / \mathrm{kg}$, i.p.). In-vivo chronoamperometric measurements of extracellular dopamine (DA) concentration were performed as previously described (Zhou et al., 1996). The recordings were taken at rates of $10 \mathrm{~Hz}$ continuously using Nafion-coated carbon-fiber working electrodes (tip $=30 \mu \mathrm{m}$; SF1A, Quanteon, Lexington, Kentucky) and a microcomputer-controlled apparatus (FAST system, Quanteon). The release of DA was measured by changes in extracellular DA concentration after microinjection of $\mathrm{KCl}$ into the striatal parenchyma. $\mathrm{KCl}$ (70 $\mathrm{mM})$ in osmolarity balanced saline $(79 \mathrm{mM} \mathrm{NaCl}$ and $2 \mathrm{mM} \mathrm{CaCl} 2$ ) was locally applied through a micropipette in the 150 to $225 \mathrm{nl}$ range. The concentration and volume of $\mathrm{KCl}$ solution in the pipette have been previously reported to induce depolarization and release of dopamine at dopaminergic nerve terminals in vivo. The working electrode and the micropipette were mounted together with sticky wax; tips were separated by $150 \mu \mathrm{m}$. The electrode/pipette assembly was lowered into striatum (AP $0-0.5 \mathrm{~mm}, \mathrm{M} / \mathrm{L} 2.5 \mathrm{~mm}$ relative to bregma and 3.5 to $7.0 \mathrm{~mm}$ below the dura). Local application of $\mathrm{KCl}$ from the micropipette was performed by pressure ejection using a pneumatic pump (BH2, Medical System). The ejected volume was monitored by recording the change in the fluid meniscus in the pipette before and after ejection using a dissection microscope.

\section{Tyrosine hydroxylase (TH) immunoreactivity}

Animals were euthanized $>2$ month after lesioning. Animals were anesthetized with chloral hydrate $(400 \mathrm{mg} / \mathrm{kg}$ i.p.) and perfused transcardially with saline followed by 
$4 \%$ paraformaldehyde (PFA) in phosphate buffer (PB; $0.1 \mathrm{M} ; \mathrm{pH}$ 7.2). The brains were dissected, post-fixed in PFA for 18-20 hours, and transferred to $18 \%$ sucrose in $0.1 \mathrm{M} \mathrm{PB}$ for at least 16 hours. Serial sections of the entire brain were cut at $40 \mu \mathrm{m}$ thickness in a cryostat. One series from every sixth section was stained for $\mathrm{TH}$. In order to control for staining variability, specimens from all experimental groups were included in every batch and reacted together in a net well tray under the same conditions. Sections were rinsed in $0.1 \mathrm{M}$ phosphate buffer $(\mathrm{PB})$, blocked with $4 \%$ bovine serum albumin (BSA) and $0.3 \%$ Triton $x-100$ in $0.1 \mathrm{M}$ PB. Sections were then incubated in a primary antibody solution mouse monoclonal anti-TH diluted in $4 \%$ BSA and $0.3 \%$ Triton $\mathrm{x}-100$ in $0.1 \mathrm{M} \mathrm{PB}$, concentration 1:100 (Chemicon, Temecula, CA) for 17-19 hours at $4^{\circ} \mathrm{C}$. Sections were then rinsed in $0.1 \mathrm{M} \mathrm{PB}$ and incubated in biotinylated horse anti-mouse IgG in the buffer (1:200; Vector Laboratories, Burlingame CA) for 1 hour, followed by incubation for 1 hour with avidin-biotinhorseradish peroxidase complex. Staining was developed with 2,3/ diaminobenzidine tetrahydrochloride $(0.5 \mathrm{mg} /$ $\mathrm{ml}$ in $50 \mathrm{mM}$ Tris- $\mathrm{HCl}$ buffer 7.4). Control sections were incubated without primary antibody. Sections were mounted on slides, and cover slipped.

\section{Stereological analysis}

The total number of $\mathrm{TH}(+)$ neurons was estimated bilaterally every $6^{\text {th }}$ section through the extent of the midbrain for adult control and 6-OHDA treated rats. The substantia nigra compacta $(\mathrm{SNc})$ was outlined under a low magnification objective $(5 \mathrm{x})$ following landmarks from the Paxinos and Watson rat atlas (Paxinos, G, 2004) and the stereologic analysis was performed under the 40x objective of a Leica DM5000B microscope (Leica Microsystems, Bannockburn, IL) and analyzed with StereoInvestigator software (MBF Bioscience, Williston, VT). The optical fractionator probe was used to generate an estimate of the total number of $\mathrm{TH}(+)$ neurons in the SNc. For each tissue section analyzed, section thickness was assessed in each sampling site and guard zones of $2.5 \mu \mathrm{m}$ were used. Systematic random sampling design was performed and generated with the following stereologic parameters: grid size: $270 \times 185 \mu \mathrm{m}$, counting frame: $100 \times 100 \mu \mathrm{m}$ and dissector height of $25 \mu \mathrm{m}$. Our criterion for counting an individual $\mathrm{TH}(+)$ neuron was the presence of its nucleus. Coefficients of error were calculated and values $<0.10$ were accepted.

\section{Additional file}

Additional file 1: Figure S1. Increase 9cRA level in brain after intranasal delivery of 9cRA. A total of 12 rats were given either 9cRA (20 ug per animal) or vehicle intranasally. Brain tissues were harvested at 1,2 and 4 hours after administration. Tissues were extracted and 9cRA levels in brain homogenates were detected by Mass spectrometry as previously described (Kane, A. Biochem J. 388,363-369, 2005). No detectable 9cRA was found in vehicle treated rats. There is a significant increase in 9cRA level at one and 2 hours after 9cRA administration $(p<0.05$, one way ANOVA). Brain 9cRA level returned to the basal level at 4 hours after administration.

\section{Abbreviations}

6-OHDA: 6-hydroxydopamine; 9cRA: 9 cis retinoic acid; (-)AO: Without antioxidants; (+)AO: With antioxidants; atRA: All-trans retinoic acid; BBB: Blood brain barrier; BMP-7: Bone morphogenetic protein-7; DA: Dopamine; GDNF: Glial cell line derived neurotrophic factor; MPP+: 1-methyl-4phenylpyridinium; PD: Parkinson's disease; PFA: Paraformaldehyde; RA: Retinoic acid; RAR: Retinoic acid receptor; RXR: Retinoid X receptor; SOD: Superoxide dismutase; TGF: Transforming growth factor; TH: Tyrosine hydroxylase; TUNEL: Terminal deoxynucleotidyl transferase dUTP nick end labeling; VM: Ventromesenphalic.

\section{Competing interests}

The authors declare that they have no competing interests.

\section{Authors' contributions}

LY and HS carried out the surgery, behavioral and electrochemical studies. $\mathrm{OD}, \mathrm{CB}, \mathrm{SY}$ and YW carried out immunohistochemical studies. EB carried out neuronal culture study. SY and YW drafted the manuscript. All authors read and approved the final manuscript.

\section{Acknowledgment}

This study was supported by NIDA IRP.

\section{Author details}

${ }^{1}$ Neural Protection and Regeneration Section, Intramural Research Program, National Institute on Drug Abuse, Baltimore, MD, USA. ${ }^{2}$ Integrative Neuroscience Section, National Institute on Drug Abuse, IRP, Baltimore, MD, USA. ${ }^{3}$ Department of Neurosurgery, Luhe Teaching Hospital, Capital Medical University, Beijing, China.

Received: 1 June 2012 Accepted: 2 October 2012

Published: 6 October 2012

\section{References}

1. Mic FA, Molotkov A, Benbrook DM, Duester G: Retinoid activation of retinoic acid receptor but not retinoid $\mathrm{X}$ receptor is sufficient to rescue lethal defect in retinoic acid synthesis. Proc Natl Acad Sci USA 2003, 100:7135-7140.

2. Horton C, Maden M: Endogenous distribution of retinoids during normal development and teratogenesis in the mouse embryo. Dev Dyn 1995, 202:312-323.

3. Ulven SM, Gundersen TE, Sakhi AK, Glover JC, Blomhoff R: Quantitative axial profiles of retinoic acid in the embryonic mouse spinal cord: 9-cis retinoic acid only detected after all-trans-retinoic acid levels are superelevated experimentally. Dev Dyn 2001, 222:341-353.

4. Werner EA, Deluca HF: Metabolism of a physiological amount of alltrans-retinol in the vitamin A-deficient rat. Arch Biochem Biophys 2001, 393:262-270.

5. Levin AA, Sturzenbecker LJ, Kazmer S, Bosakowski T, Huselton C, Allenby G, et al: 9-cis retinoic acid stereoisomer binds and activates the nuclear receptor RXR alpha. Nature 1992, 355:359-361.

6. Allenby G, Janocha R, Kazmer S, Speck J, Grippo JF, Levin AA: Binding of 9-cis-retinoic acid and all-trans-retinoic acid to retinoic acid receptors alpha, beta, and gamma. Retinoic acid receptor gamma binds all-trans-retinoic acid preferentially over 9-cis-retinoic acid. J Biol Chem 1994, 269:16689-16695.

7. Mangelsdorf DJ, Ong ES, Dyck JA, Evans RM: Nuclear receptor that identifies a novel retinoic acid response pathway. Nature 1990, 345:224-229.

8. Kitamura M, Ishikawa Y, Moreno-Manzano V, Xu Q, Konta T, Lucio-Cazana J, et al: Intervention by retinoic acid in oxidative stress-induced apoptosis. Nephrol Dial Transplant 2002, 17(Suppl 9):84-87. 
9. Boniece IR, Wagner JA: NGF protects PC12 cells against ischemia by a mechanism that requires the N-kinase. J Neurosci Res 1995, 40:1-9.

10. de Urquiza AM, Liu S, Sjoberg M, Zetterstrom RH, Griffiths W, Sjovall J, et al: Docosahexaenoic acid, a ligand for the retinoid $X$ receptor in mouse brain. Science 2000, 290:2140-2144.

11. Glozman S, Green P, Yavin E: Intraamniotic ethyl docosahexaenoate administration protects fetal rat brain from ischemic stress. $J$ Neurochem 1998, 70:2484-2491.

12. Umemura K, Toshima $Y$, Asai F, Nakashima M: Effect of dietary docosahexaenoic acid in the rat middle cerebral artery thrombosis model. Thromb Res 1995, 78:379-387.

13. Harvey BK, Shen $H$, Chen GJ, Yoshida Y, Wang Y: Midkine and retinoic acid reduce cerebral infarction induced by middle cerebral artery ligation in rats. Neurosci Lett 2004, 369:138-141.

14. Shen H, Luo Y, Kuo CC, Deng X, Chang CF, Harvey BK, et al: 9-Cis-Retinoic acid reduces ischemic brain injury in rodents via bone morphogenetic protein. J Neurosci Res 2009, 87:545-555.

15. Cheung YT, Lau WK, Yu MS, Lai CS, Yeung SC, So KF, et al: Effects of alltrans-retinoic acid on human SH-SY5Y neuroblastoma as in vitro model in neurotoxicity research. NeuroToxicology 2009, 30:127-135.

16. Tieu K, Zuo DM, Yu PH: Differential effects of staurosporine and retinoic acid on the vulnerability of the SH-SY5Y neuroblastoma cells: involvement of bcl-2 and p53 proteins. J Neurosci Res 1999, 58:426-435.

17. Katsuki H, Kurimoto E, Takemori S, Kurauchi Y, Hisatsune A, Isohama Y, et al: Retinoic acid receptor stimulation protects midbrain dopaminergic neurons from inflammatory degeneration via BDNF-mediated signaling. J Neurochem 2009, 110:707-718.

18. Ulusoy GK, Celik T, Kayir H, Gursoy M, Isik AT, Uzbay TI: Effects of pioglitazone and retinoic acid in a rotenone model of Parkinson's disease. Brain Res Bull 2011, 85:380-384.

19. Lin SZ, Hoffer BJ, Kaplan P, Wang Y: Osteogenic protein-1 protects against cerebral infarction induced by MCA-ligation in adult rats. Stroke 1999, 30:126-133.

20. Wang $Y$, Lin SZ, Chiou AL, Williams LR, Hoffer BJ: Glial cell line-derived neurotrophic factor protects against ischemia-induced injury in the cerebral cortex. J Neurosci 1997, 17:4341-4348.

21. Harvey BK, Mark A, Chou J, Chen GJ, Hoffer BJ, Wang Y: Neurotrophic effects of bone morphogenetic protein-7 in a rat model of Parkinson's disease. Brain Res 2004, 1022:88-95

22. Chou J, Luo Y, Kuo CC, Powers K, Shen H, Harvey BK, et al: Bone morphogenetic protein-7 reduces toxicity induced by high doses of methamphetamine in rodents. Neuroscience 2008, 151:92-103.

23. Chou J, Harvey BK, Ebendal T, Hoffer BJ, Wang Y: Nigrostriatal alterations in bone morphogenetic protein receptor II dominant negative mice. Acta Neurochir Supp/ 2008, 101:93-98.

24. Sabeti J, Gerhardt GA, Zahniser NR: Acute cocaine differentially alters accumbens and striatal dopamine clearance in low and high cocaine locomotor responders: behavioral and electrochemical recordings in freely moving rats. J Pharmacol Exp Ther 2002, 302:1201-1211.

25. Sabeti J, Adams CE, Burmeister J, Gerhardt GA, Zahniser NR: Kinetic analysis of striatal clearance of exogenous dopamine recorded by chronoamperometry in freely-moving rats. J Neurosci Methods 2002, 121:41-52.

26. Wang $Y$, Chang CF, Morales M, Chiang YH, Harvey BK, Su TP, et al: Diadenosine tetraphosphate protects against injuries induced by ischemia and 6-hydroxydopamine in rat brain. J Neurosci 2003, 23:7958-7965.

27. Yu SJ, Airavaara M, Shen H, Chou J, Harvey BK, Wang Y: Suppression of endogenous PPARy increases vulnerability to methamphetamine -induced injury in mouse nigrostriatal dopaminergic pathway. Psychopharmacology (Berl) 2012, 221:479-492.

28. Cass WA, Manning MW: Recovery of presynaptic dopaminergic functioning in rats treated with neurotoxic doses of methamphetamine. J Neurosci 1999, 19:7653-7660.

29. Ahlemeyer B, Bauerbach E, Plath M, Steuber M, Heers C, Tegtmeier F, et al: Retinoic acid reduces apoptosis and oxidative stress by preservation of SOD protein level. Free Radic Biol Med 2001, 30:1067-1077.

30. Ahlemeyer B, Huhne R, Krieglstein J: Retinoic acid potentiated the protective effect of NGF against staurosporine-induced apoptosis in cultured chick neurons by increasing the trkA protein expression. J Neurosci Res 2000, 60:767-778.
31. Hudson JL, van Horne CG, Stromberg I, Brock S, Clayton J, Masserano J, et al: Correlation of apomorphine- and amphetamine-induced turning with nigrostriatal dopamine content in unilateral 6-hydroxydopamine lesioned rats. Brain Res 1993, 626:167-174.

32. Hoffman AF, Lupica CR, Gerhardt GA: Dopamine transporter activity in the substantia nigra and striatum assessed by high-speed chronoamperometric recordings in brain slices. $J$ Pharmacol Exp Ther 1998, 287:487-496.

33. Bernheimer H, Birkmayer W, Hornykiewicz O, Jellinger K, Seitelberger $F$ : Brain dopamine and the syndromes of Parkinson and Huntington. Clinical, morphological and neurochemical correlations. J Neurol Sci 1973, 20:415-455.

34. Bezard E, Gross CE, Brotchie JM: Presymptomatic compensation in Parkinson's disease is not dopamine-mediated. Trends Neurosci 2003, 26:215-221.

35. Chang CF, Morales M, Chou J, Chen HL, Hoffer BJ, Wang Y: Bone morphogenetic proteins are involved in fetal kidney tissue transplantation -induced neuroprotection in stroke rats. Neuropharmacology 2002, 43:418-426.

36. Wang Y, Chang CF, Morales M, Chou J, Chen HL, Chiang YH, et al: Bone morphorgenetic protein- 6 reduces ischemia-induced brain damage in rats. Stroke 2001, 32:2170-2178.

37. Shinozaki Y, Sato Y, Koizumi S, Ohno Y, Nagao T, Inoue K: Retinoic acids acting through retinoid receptors protect hippocampal neurons from oxygen-glucose deprivation-mediated cell death by inhibition of c-jun$\mathrm{N}$-terminal kinase and p38 mitogen-activated protein kinase. Neuroscience 2007, 147:153-163.

38. Goldstein JT, Dobrzyn A, Clagett-Dame M, Pike JW, DeLuca HF: Isolation and characterization of unsaturated fatty acids as natural ligands for the retinoid-X receptor. Arch Biochem Biophys 2003, 420:185-193.

39. Bazan NG, Molina MF, Gordon WC: Docosahexaenoic acid signalolipidomics in nutrition: significance in aging, neuroinflammation, macular degeneration, Alzheimer's, and other neurodegenerative diseases. Annu Rev Nutr 2011, 31:321-351.

40. Calandria JM, Mukherjee PK, de Rivero Vaccari JC, Zhu M, Petasis NA, Bazan NG: Ataxin-1 poly-Q-induced proteotoxic stress and apoptosis are attenuated in neural cells by docosahexaenoic acid-derived neuroprotectin D1. J Biol Chem 2012, 287:23726-23739.

41. Heyman RA, Mangelsdorf DJ, Dyck JA, Stein RB, Eichele G, Evans RM, et al: 9-cis retinoic acid is a high affinity ligand for the retinoid $X$ receptor. Cell 1992, 68:397-406.

42. Liu DM, Lin SZ, Wang SD, Wu Ml, Wang Y: Xenografting human T2 sympathetic ganglion from hyperhidrotic patients partially restores catecholaminergic functions in hemi-Parkinsonian athymic rats. Cell Transplant 1999, 8:563-591

43. Luo Y, Hoffer BJ, Wang Y: Rotation, drug-induced. In The encyclopedia of movement disorders (MOVE). 1st edition. Edited by Kompoliti K, Verhagen L. London, England: Academic Press; 2010:e15193.

44. van den Berg MP, Romeijn SG, Verhoef JC, Merkus FW: Serial cerebrospinal fluid sampling in a rat model to study drug uptake from the nasal cavity. J Neurosci Methods 2002, 116:99-107

doi:10.1186/1471-2202-13-120

Cite this article as: Yin et al:: Early post-treatment with 9-cis retinoic acid reduces neurodegeneration of dopaminergic neurons in a rat model of Parkinson's disease. BMC Neuroscience 2012 13:120. 